\title{
Nonlinear Effects of Winter Swimming and Sauna Recreational Activities on the Heart Rate Variability
}

\author{
Ilya Potapov ${ }^{1}$, Samuli Haverinen ${ }^{1}$, Juhani Smolander ${ }^{2}$ Jari Viik $^{3}$, Esa Räsänen ${ }^{1}$ \\ ${ }^{1}$ Laboratory of Physics, Tampere University of Technology, Finland \\ ${ }^{2}$ Department of Design Sciences, Lund University, Sweden \\ ${ }^{3}$ Faculty of Biomedical Sciences and Engineering, Tampere University of Technology, Finland
}

\begin{abstract}
Sauna sessions and winter swimming are traditional and popular recreational activities in certain countries. Their positive effects on health and relaxation, both as separate and combined activities, are commonly reported. However, systematic studies of these effects are relatively scarce, especially regarding the nonlinear analysis of the physiological measurements of the heart activity.

We performed Multi-Scale Entropy (MSE) and Detrended Fluctuation (DFA) analyses on the inter-beat time series (about $72 \mathrm{~h}$ long) of 21 healthy volunteers studied in three distinct contexts: winter swimming combined with sauna bathing $(W)$, sauna bathing alone $(S)$, and control (C) with no related activities.

We confirmed that the scaling exponents (DFA) and complexity indices (determined from MSE) stay within the variation observed for healthy individuals as compared to public data sets. Next, we showed that $S$ and $W$ interventions have uncorrelated effects on the whole time series complexity in each individual. Additionally, the long-range scaling properties of $S$ and $W$ groups are not correlated as determined by DFA. Thus, we speculate that winter swimming combined with sauna bathing and sauna bathing alone might have different physiological responses.
\end{abstract}

\section{Introduction}

Sauna bathing, alone or combined with winter swimming, is a traditional and popular recreational activity in Northern countries, especially Finland. Sauna sessions and winter swimming are believed to relieve the work-related stress, to refresh mind, and to improve quality of sleep [1,2].

Previous studies of these activities focused on time and frequency domain characteristics of the inter-beat time series [3]. The aim of this study is to analyze nonlinear scaling properties of the inter-beat dynamics of the heart during sauna and winter swimming interventions.

\section{Data and methods}

Data were collected from 27 volunteers (16 females, 11 males) with the following criteria for each of them:

- the subject is an experienced winter swimmer, regularly combining sauna bathing and winter swimming;

- the subject's age is between 35 to 55 years;

- the subject has a normal day time work;

- the subject has normal health status and no regular medications;

- the subject has no sleep disorders, nor severe snoring;

- the subject has a normal body-mass index (BMI) and regular living habits.

The measurement protocol consisted of three independent trials for each of the subject: sauna bathing (S), ice-cold water immersion/winter swimming combined with sauna (W), and control trial without any intervention (C). Each trial took place on weekdays and lasted for three consecutive days: the heart rate measurements started in the morning of the first day and stopped in the morning of the fourth day. During intervention trials $\mathrm{S}$ and $\mathrm{W}$, only the first two days included the corresponding exposure (also subjects were instructed to take the exposures $\mathrm{S} / \mathrm{W}$ after work in the evening), the third day was left without the exposure. In the reference trial subjects were forbidden to take sauna bathing or swim in ice-cold water.

Heart rate was recorded continuously during all three days for each trial using an ambulatory recorder belt (Suunto Memory Belt, Suunto, Vantaa, Finland, www.suunto.com). In this study we use consecutive RR (inter-beat) intervals of the ECG recordings as these reveal long-range scaling properties [4] that were shown using nonlinear techniques from statistical physics. The inter-beat time series were analyzed using two methods: Multi-Scale Entropy (MSE) [5, 6] and Detrended Fluctuation (DFA) [4, 7] analyses.

The MSE analysis allows for quantifying a complexity of a time series by calculating entropy (here, Sample 
Entropy $[8,9])$ over different scales of the time series. The Sample Entropy (SampEn) is a regularity statistic quantifying how regular is the pattern of a time series. The scale of a time series is determined by the coarsegraining procedure: the data points from the original time series are averaged within non-overlapping windows of increasing length with scale being the length of the windows (see $[5,6]$ for details).

It was proposed to use sum of SampEn values over a range of scales as a complexity index $\mathrm{CI}[10,11]$. $\mathrm{CI}$ is an integrated measure, accounting for variation in the time series at multiple scales. In this work we use the first 10 scales of a time series to calculate CI.

The time series were preprocessed to remove artifact regions. Only 21 subjects with all three trials were considered for the analysis. In permutation tests, $10^{5}$ permutations were used to estimate $p$-values.

\section{Results}

A typical three-day time series of the RR intervals is shown in Fig. 1. Note the highly regular pattern for the W intervention (Fig. 1C): the valleys of low RR correspond to the high frequency activity of the heart during sauna sessions, whereas the peaks of high RR correspond to staying in cold open air (90-95\% of the time) and in icecold water during winter swimming sessions. This fact prompts us to question whether such patterns have an effect on the properties of the whole time series.

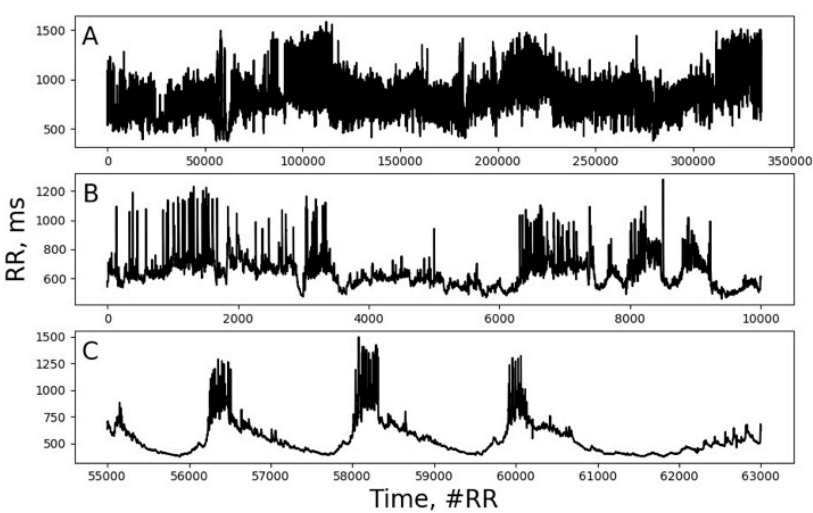

Figure 1. A typical time series of RR intervals (W trial). (A) full time series approximately $72 \mathrm{~h}$ long; (B) a short fragment of the full series; (C) a close-up corresponding to the sauna bathing and winter swimming activity.

\subsection{Multi-Scale Entropy analysis}

After removing all artifacts we have performed the MSE analysis over full time series. The results are shown in Fig. 2. From the figure one can see that there is no significant variation in the form of the MSE curves and in the span of SampEn values across the three trials. We have verified using publicly available data (PhysioNet [12]) that healthy individuals have similar shape of the curves (Fig. 2A).
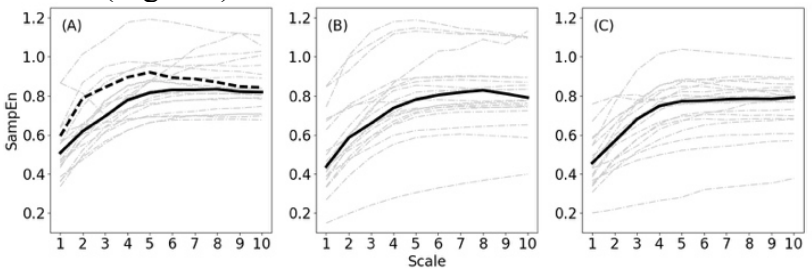

Figure 2: sample entropy (SampEn) values over the first 10 scales of the original time series. (A) control, C; (B) winter swimming and sauna, W; (C) sauna bathing, S. The solid black line denotes the median profile from all the curves. The dash black line denotes a median MSE profile from 5 time series (approximately $24 \mathrm{~h}$ long) for healthy individuals from public data base PhysioNet [12]. Scaling of the axes is the same for all three plots.

The CI distributions do not reveal overall differences (Table 1). However, if we perform statistical tests between Cl's of the three groups we find significant difference between $\mathrm{C}$ and $\mathrm{S}$ groups $(\mathrm{p}=0.031$, Table 2$)$.

Table 1. Distribution statistics of CI's for all groups.

\begin{tabular}{llll}
\hline Statistics & C group & S group & W group \\
\hline Mean & 7.76 & 6.92 & 7.50 \\
\hline Median & 7.58 & 7.28 & 7.40 \\
\hline Stand. dev. & 1.17 & 1.33 & 1.81 \\
\hline Max & 11.03 & 9.34 & 10.92 \\
\hline Min & 6.09 & 2.92 & 2.99 \\
\hline
\end{tabular}

Table 2. Statistical comparison of CI's between the three trials. Paired t-test was used. * - significant difference with the false positive rate 0.05 .

\begin{tabular}{llll}
\hline Statistics & $\mathrm{C}-\mathrm{W}$ & $\mathrm{C}-\mathrm{S}$ & $\mathrm{W}-\mathrm{S}$ \\
\hline $\mathrm{t}$-statistic & 0.708 & 2.321 & 1.096 \\
\hline $\mathrm{p}$ value & 0.487 & $0.031^{*}$ & 0.286 \\
\hline
\end{tabular}

Finally, to appreciate the by-person change in complexities between trials we present the following graph. We construct the difference measure between CI's, namely, $\delta \mathrm{C}_{\mathrm{XY}}=\mathrm{CI}(\mathrm{X})-\mathrm{CI}(\mathrm{Y})$, and plot $\delta \mathrm{C}_{\mathrm{XY}}$ vs. $\delta \mathrm{C}_{\mathrm{XZ}}$. The graph shows the complexity change when passing from a trial $\mathrm{X}$ to a trial $\mathrm{Y}$ as a function of the change between the trial $\mathrm{X}$ and a third trial $\mathrm{Z}$.

We observe a strong linear relationship between the $\delta \mathrm{C}_{\mathrm{WC}}$ and $\delta \mathrm{C}_{\mathrm{Ws}}$ (Pearson's $\rho=0.73$, two-sided permutation test $\mathrm{p}=0.0004$ ) and between $\delta \mathrm{C}_{\mathrm{SC}}$ and $\delta \mathrm{C}_{\mathrm{Sw}}(\rho=0.71, \mathrm{p}=0.0004)$ in Fig $3 \mathrm{~A}$ and $\mathrm{B}$, respectively. The $\mathrm{W}$-to-C change in $\mathrm{CI}$ is highly correlated with the $\mathrm{W}$-to-S change for a subject, which indicates that $\mathrm{C}$ and $\mathrm{S}$ trials cause a comparable (with a 
factor of $\rho$ on average) complexity change effect when applied to the $\mathrm{W}$ trial (Fig. 3A). Similarly, the W and C trials cause a correlated effect when applied to the $\mathrm{S}$ trial on per-subject basis (Fig. 3B). However, we can see that the $\mathrm{W}$ and $\mathrm{S}$ interventions are not correlated $(\rho=-0.03, \mathrm{p}$ $=0.8601)$ for each subject when applied to the $\mathrm{C}$ trial (Fig. 3C). In other words, winter swimming combined with sauna bathing and sauna bathing alone have unrelated complexity effects for each individual.
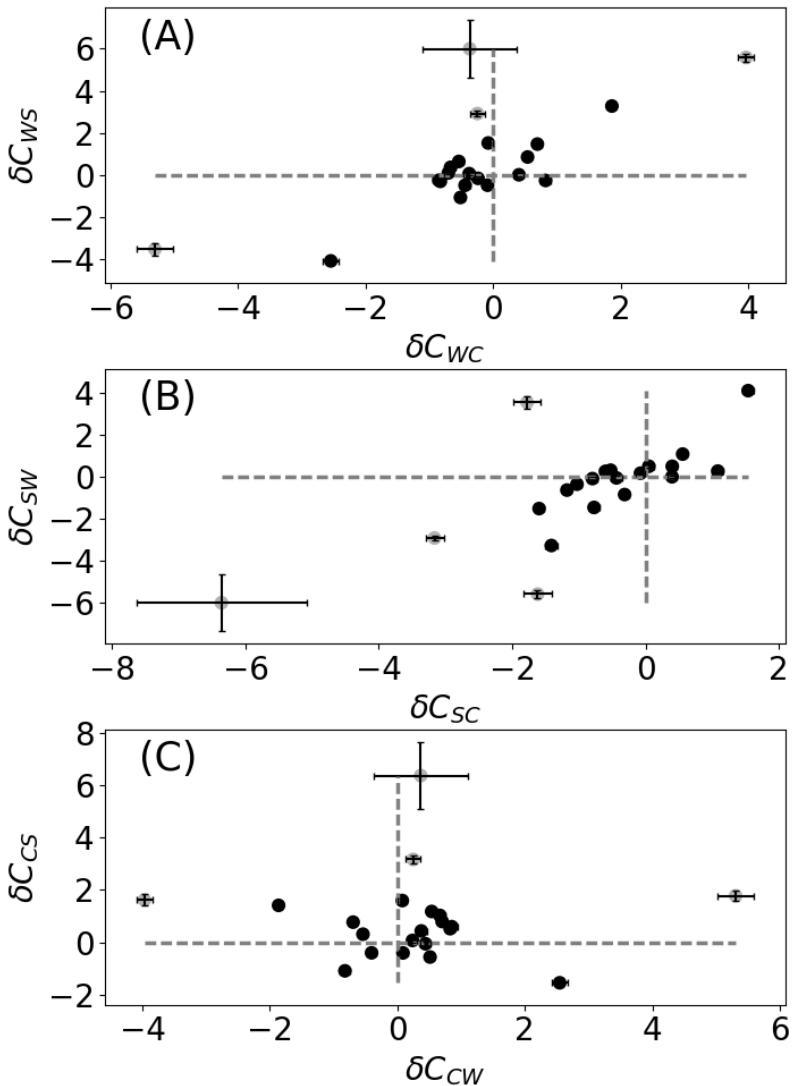

Figure 3: complexity difference plot. Subjects with more than $40 \%$ of artifact recordings in at least one of the three trials are shown in gray. Pearson correlation coefficients between data in $\mathrm{X}$ - and Y-axis: (A) $\rho=0.73$ $\mathrm{p}=0.0004$; (B) $\rho=0.71, \mathrm{p}=0.0004$; (C) $\rho=-0.03, \mathrm{p}=$ 0.8601 .

If we remove subjects with high percentage $(>40 \%)$ of artifacts (gray in Fig. 3), the correlation coefficients become: $\rho=0.83(p=0.0005), \rho=0.80\left(p=10^{-5}\right), \rho=$ $-0.34(p=0.1855)$ for the cases $A, B$, and C in Fig. 3, respectively, which indicates that the effects persist.

If we divide the plane by the coordinate axes (dashed gray lines), we also note that the southeast quarter in Fig. $3 \mathrm{~B}$ is less populated (1 point) than others. This corresponds to a less number of individuals for whom the following inequality holds $\mathrm{CI}(\mathrm{W})>\mathrm{CI}(\mathrm{S})>\mathrm{CI}(\mathrm{C})(\mathrm{p}=$ $0.019)$.

\subsection{Detrended Fluctuation analysis}

Additionally, we have estimated scaling exponents by means of DFA [7] on the whole time series in order to determine whether the scaling properties of the inter-beat time series break for the given interventions. Namely, we assessed the scaling exponents $\alpha_{1}$ (in window size $\mathrm{s}=$ $4, \ldots, 16), \alpha_{2}(\mathrm{~s}=32, \ldots, \mathrm{N} / 4$, where $\mathrm{N}$ is the total length of a time series $), \alpha_{3}(\mathrm{~s}=16, \ldots, 128)$. Note that $\alpha_{1}$ corresponds to short-range correlations, whereas $\alpha_{2}$ and $\alpha_{3}$ correspond to long-range correlations.

As with MSE, we observe the exponents' values within ranges characterizing the healthy heart dynamics. Nevertheless, we found strong correlations between $\alpha_{1}$ values for all experimental groups. Namely, Pearson's $\rho_{\mathrm{CW}}=0.86$ for $\alpha_{1}$ exponents between $\mathrm{C}$ and $\mathrm{W}$ groups, similarly $\rho_{\mathrm{CS}}=0.90$ and $\rho_{\mathrm{SW}}=0.86$ (two-sided permutation test $p$-value for Pearson's $\rho$ in all three cases was $<10^{-5}$ ). This suggests the short-range correlations of the inter-beat dynamics are not affected by any of the interventions. However, long-range correlations break between the trials. Thus, for $\alpha_{2}$ exponents: $\rho_{\mathrm{CW}}=0.33(\mathrm{p}=$ $0.15), \rho_{\mathrm{CS}}=0.48(\mathrm{p}=0.03), \rho_{\mathrm{SW}}=0.30(\mathrm{p}=0.18)$. Noteworthy, the Pearson correlation between $\alpha_{2}$ values for $\mathrm{S}$ and $\mathrm{W}$ groups also breaks suggesting the interventions have different long-range effects on the inter-beat dynamics.

The scaling exponent $\alpha_{3}$ on the window span of up to 128 beats demonstrates strong correlation between the trials, indicating that this range is not sufficient to break the inter-beat correlations $\left(\rho_{\mathrm{CW}}=0.92, \rho_{\mathrm{CS}}=0.81, \rho_{\mathrm{SW}}=\right.$ $0.90, \mathrm{p}<10^{-5}$ for all).

\section{Discussion}

We have conducted the analysis of the whole time series from 21 individuals whose heart activity was recorded during winter swimming and sauna bathing sessions. In this work, we have focused on the nonlinear analysis of time series, namely, Multi-Scale Entropy and Detrended Fluctuation analyses.

Although sauna bathing and winter-swimming RR time series exhibit a specific pattern (Fig. 1C), the whole time series analysis showed that the variation stays within physiological limits observed in healthy individuals.

However, in each particular individual winter swimming combined with sauna (W) and sauna bathing alone (S) have uncorrelated effects on the complexity of the whole time series as determined by the MSE analysis. This might indicate separate physiological mechanisms involved in the heart rate control during these two interventions. This result seems to be particular interesting given the two interventions have a common activity, that is sauna bathing, and given that we have not found any correlation with other characteristics of the 
subjects, like gender, age, BMI, health index, stress and relaxation levels etc.

Finally, we have found that the long-range correlations of the heart inter-beat dynamics break between the experimental groups as determined by the DFA scaling exponents. Short-range correlations do not break indicating that the specific winter swimming and sauna pattern of the heart's activity (Fig. 1C) cannot interfere the short scale properties of the inter-beat dynamics. However, on the large scale the activity pattern has a direct effect on the fluctuation levels defined in DFA [4, 7] and, hence, on the scaling exponents of the time series. Moreover, the large-scale properties in $\mathrm{S}$ and $\mathrm{W}$ groups are not correlated; suggesting, as in the case of the MSE results, that sauna activity alone and sauna combined with winter swimming have different physiological effects on the heart rate dynamics.

\section{References}

[1] Viik J and Smolander J. Sauna, winter swimming and perceived well-being. In: XV International sauna congress 27.-28.5.2010, Tokyo, Japan, 2010:71-72.

[2] Huttunen P, Kokko L, Ylijukuri V. Winter swimming general well-being. International Journal of Circumpolar Health 2004;63:140-44.

[3] Viik J, Rajala S, Riikonen R, Heinonen R, Nygård $\mathrm{CH}$, Perttunen J, Smolander J. Effects of sauna and winter swimming on heart rate variability, quality of sleep and perceived well-being. In: 4th International Hyvite Symposium on Wellbeing Technology, 9.6.2010, Tampere, Finland, 2010:30-31.

[4] Peng CK, Havlin S, Stanley HE, Goldberger AL. Quantification of scaling exponents and crossover phenomena in nonstationary heartbeat time series. Chaos 1995;5:82-7.
[5] Costa M, Goldberger AL, Peng CK. Multiscale entropy analysis of physiological time series. Phys Rev Lett 2002;89:062102.

[6] Costa M, Goldberger AL, Peng CK. Multiscale entropy analysis of biological signals. Phys Rev E 2005;71:021906.

[7] Peng CK, Buldyrev SV, Havlin S, Simons M, Stanley HE, Goldberger AL. Mosaic organization of DNA nucleotides. Phys Rev E 1994;49:1685-89.

[8] Richman JS, Moorman JR. Physiological time series analysis using approximate entropy and sample entropy. Am J Physiol 2000;278:H2039-H2049.

[9] Lake DE, Richman JS, Griffin MP, Moorman JR. Sample entropy analysis of neonatal heart rate variability. Am J Physiol 2002;283:R789-R797.

[10] Zhang YC. Complexity and $1 / \mathrm{f}$ noise. A phase space approach. J Phs I Fr 1991;1:971-7.

[11] Costa M, Goldberger AL. Generalized Multiscale Entropy Analysis: Application to Quantifying the Complex Volatility of Human Heartbeat Time Series. Entropy 2015; 17:1197-1203.

[12] Goldberger AL, Amaral LAN, Glass L, Hausdorff JM, Ivanov PCh, Mark RG, Mietus JE, Moody GB, Peng CK, Stanley HE. PhysioBank, PhysioToolkit, and PhysioNet: Components of a New Research Resource for Complex Physiologic Signals. Circulation 2000;101(23):e215-e220.

Address for correspondence.

Name: Ilya Potapov, $\mathrm{PhD}$

Full postal address: Laboratory of Physics, Tampere University of Technology, P.O. 553, 33101 Tampere, Finland

E-mail address: ilya.potapov@tut.fi 